\title{
The handling and sampling of radical prostatectomy specimens for reporting and research: the Oxford approach
}

\author{
Pelvender Singh Gill, ${ }^{1}$ lan S D Roberts, ${ }^{2}$ Lisa Browning, ${ }^{1}$ Ranmith Perera, ${ }^{2}$ \\ Anne Y Warren, ${ }^{3}$ Freddie C Hamdy, ${ }^{4}$ Clare Verrill ${ }^{1}$
}

${ }^{1}$ Department of Cellular Pathology and Oxford Biomedical Research Centre, Oxford University Hospitals NHS Trust, Oxford, UK. ${ }^{2}$ Department of Cellular Pathology, Oxford University Hospitals NHS Trust, Oxford, UK.

${ }^{3}$ Cambridge University Hospitals NHS Foundation Trust, Cambridge, UK. ${ }^{4}$ Nuffield Department of Surgical Sciences, University of Oxford, UK

\section{Correspondence to}

Dr Clare Verrill,

Department of Cellular Pathology, Oxford University Hospitals NHS Trust, John Radcliffe Hospital, Headley Way, Oxford OX3 9DU, UK Clare.Verrill@OUH.nhs.uk

Accepted 26 June 2012 Published Online First 7 August 2012

\section{ABSTRACT}

This article reviews previously described methods of fresh tissue sampling from radical prostatectomy specimens for research and describes a method used in Oxford which is simple, logical and cost effective. The method utilises a systematic zonal approach to tissue procurement in order to meet the increasing requirement for research samples with detailed morphological information such as zone of origin, tumour stage and Gleason grade.

The described method involves punch biopsy sampling from a $4 \mathrm{~mm}$ thick transverse slice cut $8 \mathrm{~mm}$ superior to the apex. 9 biopsies are taken from each specimen in the following zonal distribution: Mid anterior, right lateral, right peripheral zone lateral, right peripheral zone mid, left peripheral zone mid, left peripheral zone lateral, left lateral, left transition zone and right transition zone.

The method was validated by successfully sampling tumour in 7 out of 8 cases (88\%). In 6 of the positive cases, tumour was present in more than 1 punch biopsy. The mean time from receipt of the specimen to completion of the biopsy freezing was 23.5 minutes. Tumour stage, zone and Gleason grade were determined for all positive biopsies. All cases were reported to RCPath guidelines with no compromising of margins.

A logical systematic method of fresh tissue sampling from radical prostatectomy specimens is presented, which balances the need for accurate routine histopathological reporting with the requirement for increasingly complex research samples to be taken with attention to morphological details such as zone and stage.

\section{INTRODUCTION}

In an era of rapidly developing advances in the field of cancer biology and genetics, it is of paramount importance that research into prostate cancer keeps pace with that of other organ systems as it is the commonest cancer arising in men in the UK. ${ }^{1}$ Regrettably, this is not the case and many aspects of the basic mechanisms by which prostate cancer arises remain elusive, by contrast to other tissues where exciting developments have translated into new management options for patients. For example, in breast cancer clear molecular subgroups have been identified with distinct clinical outcomes. ${ }^{2}$ In the treatment of malignant melanoma, the discovery that $50 \%$ of cases have activating BRAF mutations has revolutionised treatment of metastatic melanoma, which can be targeted with BRAF inhibitors such as Vemurafenib, and this drug is showing unprecedented improvement in survival outcome. ${ }^{3}$ Such exciting advances with real clinical impact are yet to materialise for prostate cancer.
Fundamental to achieving similar advances in prostate cancer is for researchers to be provided with good-quality fresh prostate tissue that can be snap frozen for DNA, RNA and protein analysis. Only limited studies can be performed on formalin-fixed paraffin-embedded tissue. Radical prostatectomies are the best source of prostatic carcinoma for research as prostate biopsies provide only limited material, while transurethral resection of prostate chippings are fraught with logistical problems including diathermy artefact and the majority of cases being benign.

There is increasing interest in the heterogeneity within and between tumours, making detailed morphological assessment of key importance. Gerlinger et $a l^{4}$ recently described the marked variation in genetic expression signatures between biopsy samples taken from different regions of renal cell carcinomas. In the prostate, it is now recognised that there are biological and genetic differences between transition zone, peripheral zone and anterior predominant tumours. ${ }^{5} 6$

Many techniques for obtaining fresh tissue from radical prostatectomy specimens have been described in the literature, with usually either a whole slice being taken for research or tissue being harvested from a cut surface, by shave sections or punch biopsy. ${ }^{7}$ Some methods require specialised equipment developed locally in the research institutions, which are not widely available. ${ }^{8-10}$ Others involve disrupting slices and subsequently the capsule and margins of the prostate, ${ }^{11}{ }^{12}$ thus compromising routine histopathological reporting. Some techniques can only be performed with macroscopically evident tumour ${ }^{11} 1314$ and as a high proportion of prostate cancers are not visible at dissection, sampling needs to be systematic rather than dependent on finding a macroscopically visible lesion. ${ }^{15}$ Methods involving trucut needle biopsies taken through the capsule may not provide sufficient material for study. ${ }^{16}$ Other techniques involve removal of large amounts of tissue for research, some methods taking 40-50\% of the prostate for research, tissue that is effectively lost from routine reporting. ${ }^{12} 17$

A simple method for systematic sampling of fresh radical prostatectomy specimens is presented in this paper, which strikes a balance between the need for uncompromised histological reporting to the standards of the RCPath guidelines ${ }^{18}$ and providing research samples with detailed morphological information such as zone, Gleason grade and tumour stage. The method is compared with previously described techniques for comparison. 


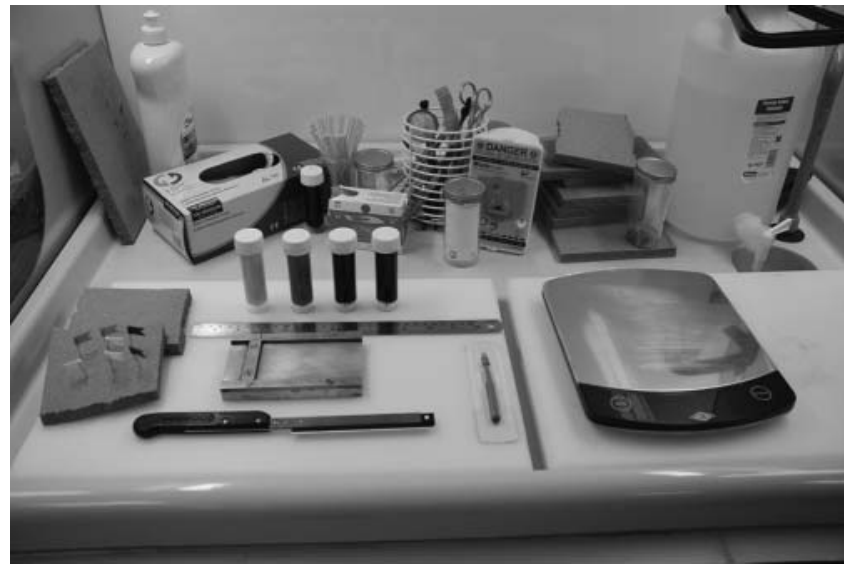

Figure 1 The basic kit required for sampling, including cutting guide plate, scales, knife, cork board, punch biopsy and a ruler. This figure is only reproduced in colour in the online version.

\section{DESCRIBED METHOD}

Patients are consented for tissue harvesting for the ProMPT study (NRES reference 01/4/061). Patients with only one positive core in the diagnostic biopsy are excluded in order to reduce the chances of a negative radical prostatectomy specimen. Patients with a prostatic volume on ultrasound of $<30 \mathrm{cc}$ and $>100 \mathrm{cc}$ are excluded because of the hypertrophy associated with larger cases (distorting the specimen when freshly sliced) and the increased chance of a negative radical prostatectomy with small prostates.

Oxford Radcliffe Biobank staff and the biobanking pathologist are notified approximately $30 \mathrm{~min}$ prior to the prostate being removed from the patient. The basic kit is prepared (figure 1): standard laboratory knife, laboratory inks, cutting guide plate with $4 \mathrm{~mm}$ cutting height and $5 \mathrm{~mm}$ skin punch biopsy utensil (Meditech Systems Ltd, Dorset, UK).

The prostate is placed in a plastic bag on ice in the operating room and transferred to pathology. The prostate is orientated, photographed and the external fascia and connective tissues are examined together with identification of any capsular incisions.

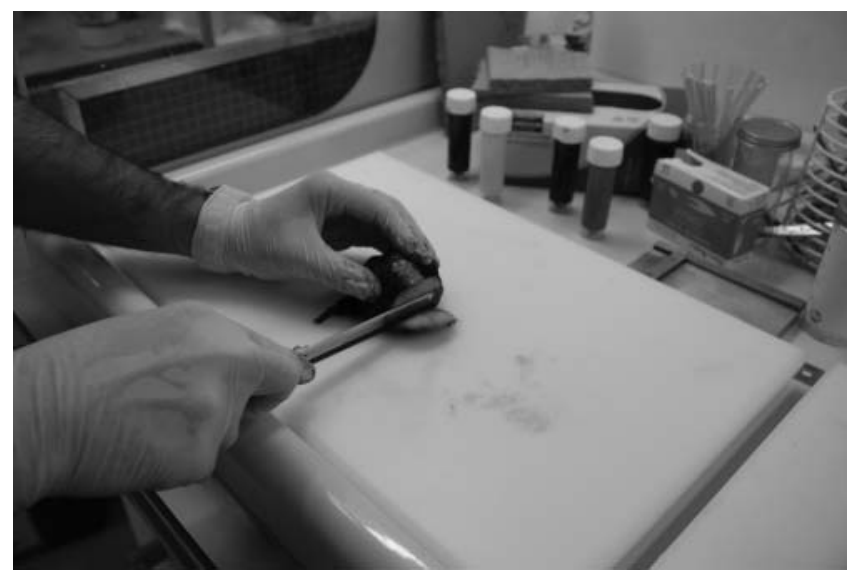

Figure 2 The prostate is orientated and capsular defects noted. The specimen is photographed front and back for documentation. After applying standard orientation inks, for example, left=black, right=blue and a strip of yellow for anterior, the prostate is placed on its relatively flat posterior surface and the apex is amputated aiming to remove a cone of approximately $8 \mathrm{~mm}$ of apical tissue in a single smooth movement with the blade. This figure is only reproduced in colour in the online version.

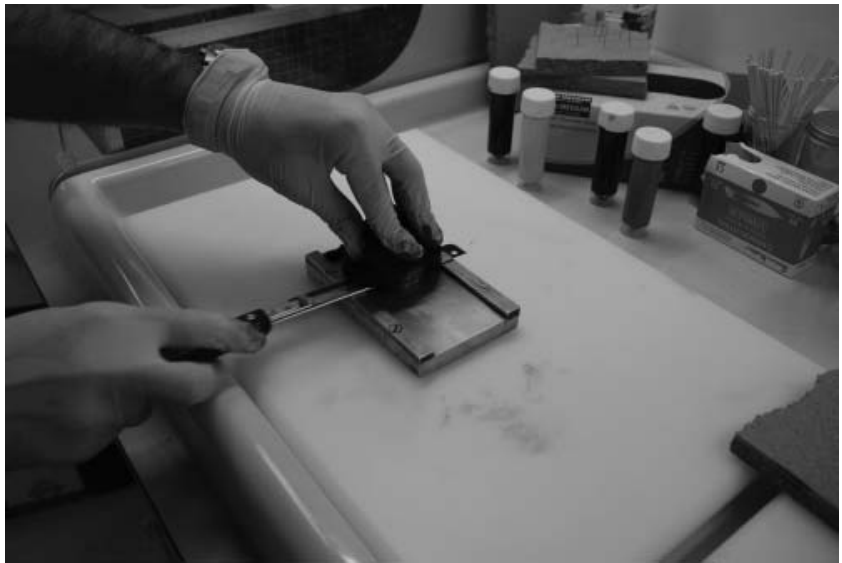

Figure 3 On removal of the apex, the organ is placed on the cutting guide plate, the newly exposed cut surface flat against the surface of the plate. The time taken will affect tissue expansion and this should be minimised and completed within 2-3 min at most (usually a minute). This figure is only reproduced in colour in the online version.

The specimen is inked according to the protocol in Oxford (right half of prostate inked blue, left half black and a strip of yellow ink marking anterior). The specimen is weighed and measured in three dimensions and seminal vesicles and vasa are also measured.

Using a standard laboratory knife, the apex of the prostate is removed approximately $8 \mathrm{~mm}$ above the urethral apex (figure 2). The cut surface of the prostate is then placed immediately onto the cutting guide plate and a $4 \mathrm{~mm}$ transverse slice is cut (figures 3 and 4). Punch biopsies are then sampled from this slice (figure 5) in the following systematic order (figure 6) and are immediately snap frozen:

1. Mid anterior

2. Right lateral

3. Right peripheral zone lateral

4. Right peripheral zone mid

5. Left peripheral zone mid

6. Left peripheral zone lateral

7. Left lateral

8. Left transition zone

9. Right transition zone.

In addition to this set of standard biopsies, additional punch biopsies can be taken if required or if the prostate is large, and the position of these biopsies is marked on a sampling diagram.

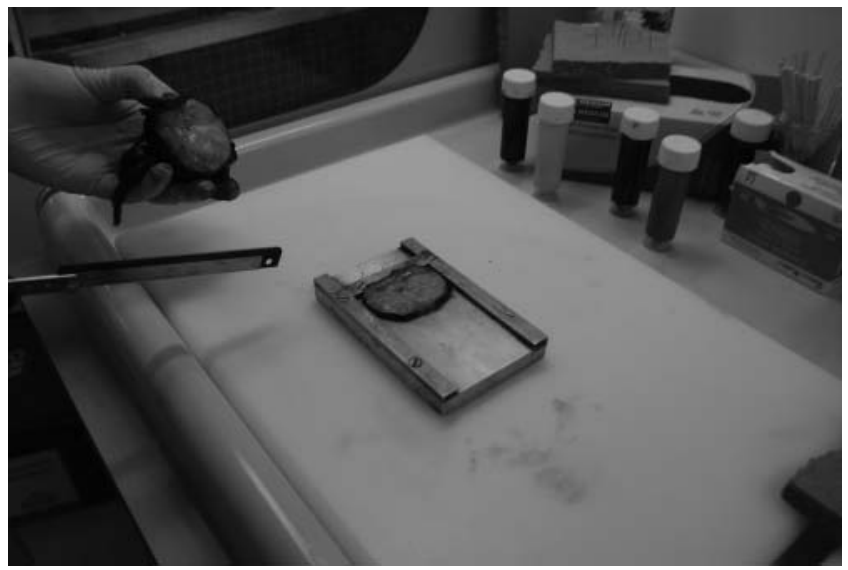

Figure 4 A $4 \mathrm{~mm}$ single slice is taken using the cutting guide plate. This figure is only reproduced in colour in the online version. 


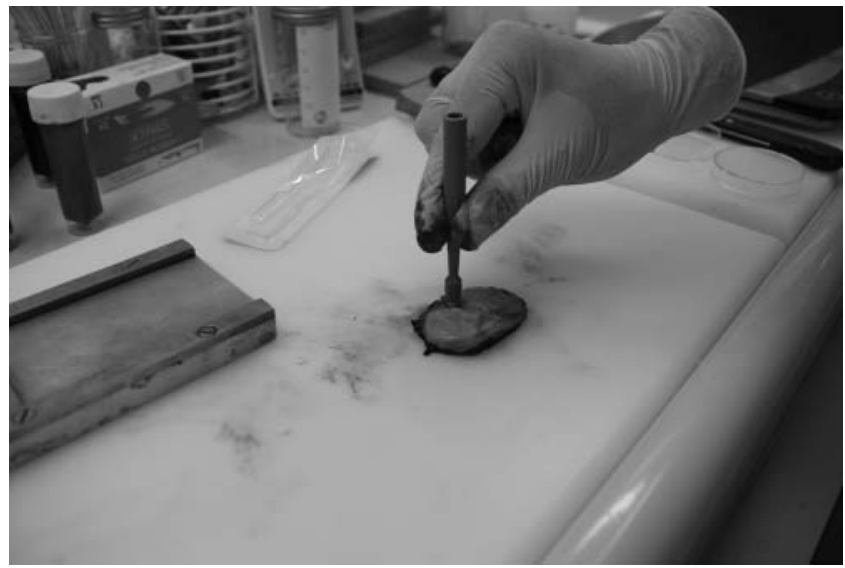

Figure 5 The slice is sampled, taking biopsies from the zones in a fixed and systematic fashion as shown in figure 6. This figure is only reproduced in colour in the online version.

Punch biopsies 1-7 are taken close to the capsule in order to maximise the chances of sampling tumour, but within the capsule to avoid compromising capsular morphology. Biopsies 2 and 7 are usually taken from the most anterior horns of the right and left peripheral zones if possible, but sometimes may be taken from the transition zone (depending on the individual prostate). The apical cone, sampled transverse slice and the rest of the prostate are then pinned to cork board, face down to minimise bulging. It is necessary to pin the capsule with care, drawing it down with pins to ensure it does not retract (figure 7). Punch biopsy holes are inked around their periphery to distinguish them from artefacts.

The prostate is then placed in formalin for fixation and subsequent routine histopathological cut up. After fixation, the sampled slice is embedded in its entirety as a whole mount (figure 8). The apical cone is sliced perpendicular to the cut surface. The main part of the prostate is sliced in complete

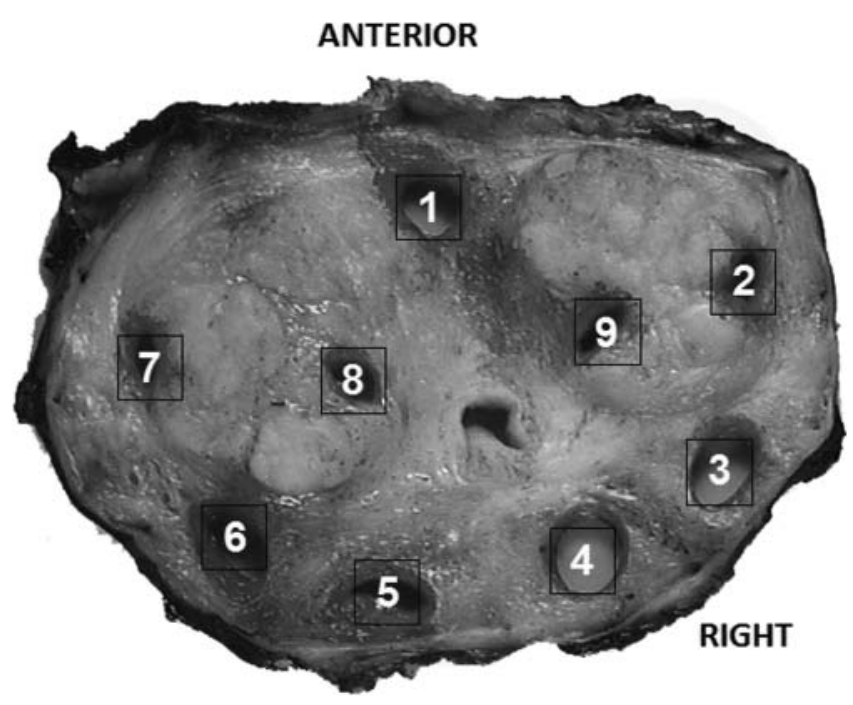

Figure 6 The slice after all sampling is complete. Punch biopsies taken as follows: (1) mid anterior, (2) right lateral, (3) right peripheral zone lateral, (4) right peripheral zone mid, (5) left peripheral zone mid, (6) left peripheral zone lateral, (7) left lateral, (8) left transition zone and (9) right transition zone. The punch biopsy sites are inked in red to distinguish these areas from artefactual defects after processing. This figure is only reproduced in colour in the online version.

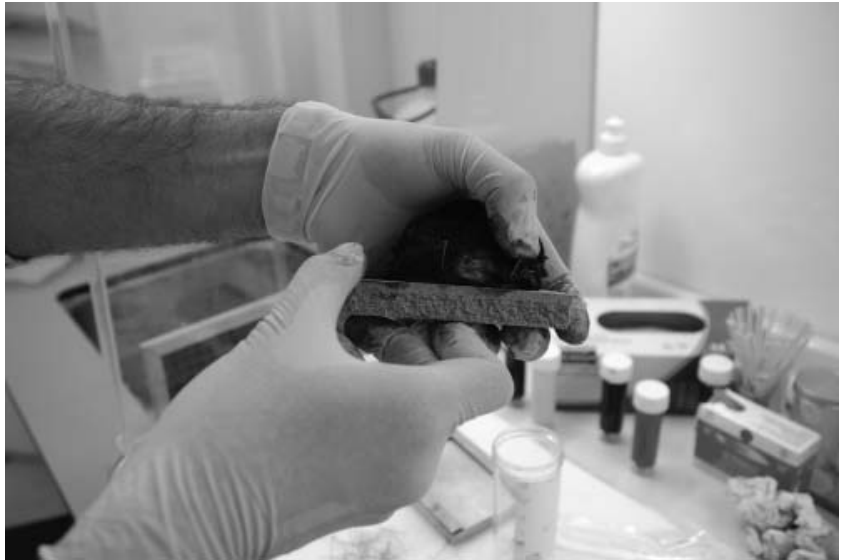

Figure 7 The prostate, apex and sampled slice are pinned to cork board. There will be some expansion of the prostate, while samples are taken. The capsule and tissue can be 'eased down' by the use of pins to ensure good contact with a relative flat surface on the cork board. This figure is only reproduced in colour in the online version.

transverse sections with the base cut as a cone and the seminal vesicles and vasa removed and embedded separately.

After routine histopathological reporting, a punch biopsy sampling table is completed, which records (from assessment of tissue around the perimeter of the biopsy site) benign/malignant tissue, punch biopsy site, Gleason pattern(s) and stage of the tumour. By entering this information onto our database, there is easy retrieval of biopsies with particular features. Any biopsies selected for research can have a frozen section cut later for absolute confirmation of tissue diagnosis.

\section{VALIDATION OF THE METHOD}

Eight cases were sampled to validate the method. There was a mean time from receipt of specimen to snap freezing of samples of $23.5 \mathrm{~min}$. Tumour was banked in $7 / 8$ cases $(88 \%)$ (table 1).

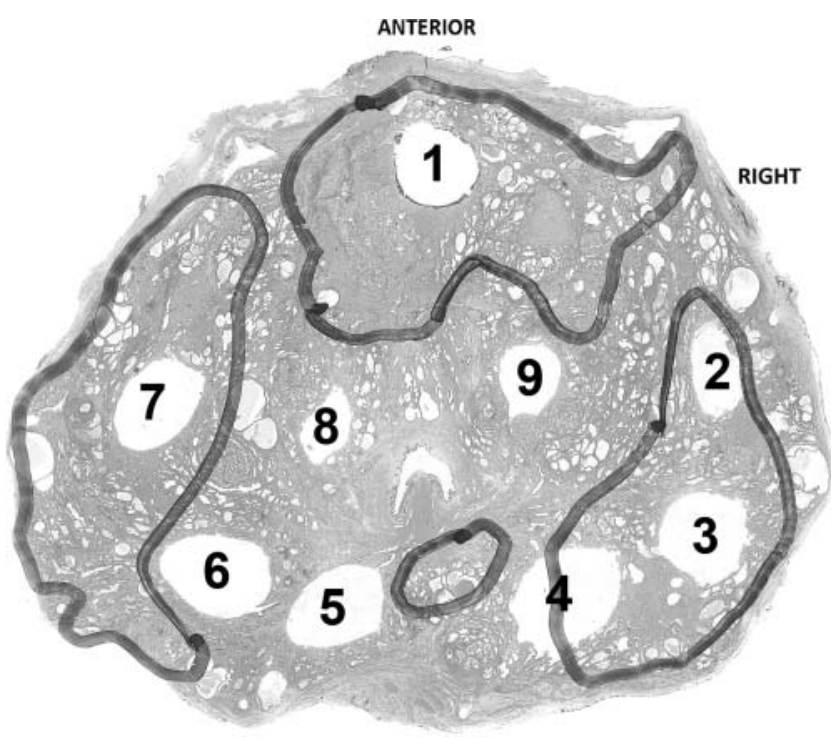

Figure 8 The sampled slice after fixation and processing. Tumour is outlined in black and five of the eight punch biopsies have sampled tumour. This figure is only reproduced in colour in the online version. 
Table 1 Details of the eight sampled radical prostatectomies with time taken for sampling (receipt of specimen to snap freezing of samples), number of punch biopsies taken and number of punch biopsies containing tumour

\begin{tabular}{lll}
\hline $\begin{array}{l}\text { Time taken for } \\
\text { sampling }\end{array}$ & $\begin{array}{l}\text { Number of punch } \\
\text { biopsies taken }\end{array}$ & $\begin{array}{l}\text { Number of punch biopsies } \\
\text { containing tumour }\end{array}$ \\
\hline 22 & 8 & 2 \\
21 & 9 & 0 \\
23 & 9 & 2 \\
25 & 9 & 2 \\
22 & 9 & 5 \\
25 & 9 & 2 \\
23 & 9 & 5 \\
24 & 9 & 1 \\
\hline
\end{tabular}

\section{DISCUSSION}

This method utilises a systematic zonal sampling approach, sampling transitional zone, peripheral zone and anterior zone. There is increasing recognition that there are biological and genetic differences between tumours arising in different zones of the prostate. For example, there are higher rates of TMPRSS2-ERG gene fusion in peripheral zone tumours compared with transition zone tumours. ${ }^{5}$ Hence, morphological identification of the punch biopsy site is of paramount importance.

Wheeler and Lebovitz ${ }^{19}$ describe a radical prostatectomy sampling method in which the prostate is divided into two halves and then 10 samples are taken using a set protocol. Four of these are from the peripheral zone, although they are taken as two mirror image pairs, effectively meaning they are only sampling two peripheral zone sites (where most tumours are likely to be). Two samples are seminal vesicle and four are from the transition zone. No anterior samples are taken. Our method samples the anterior zone and provides six biopsies from different areas in the peripheral zone and two from the transition zone. Seminal vesicle sampling is not undertaken in our method as patients with seminal vesicle invasion are usually identified on imaging and not offered surgery.

This method represents a modification of a technique previously reported by one of the co-authors (AYW) in which fresh tissue was removed from a single slice from 150 radical prostatectomy specimens, using a systematic punch sampling method, with a $76 \%$ success rate for obtaining tumour tissue. This yielded high-quality DNA and RNA extractions for genomic studies, validating this methodology for fresh tissue collection. $^{20}$

The method described in this paper is systematic and therefore tumour does not need to be macroscopically evident. The technique is quick with a mean time of $23.5 \mathrm{~min}$ from receipt of the prostate to snap freezing of samples. The protocol requires no specialist equipment, just basic laboratory items such as a sharp knife and inks, making the technique accessible. A guide plate is used for slice cutting in Oxford, as recommended in the RCPath dataset. ${ }^{18}$

The method described by Jhavar et $a l^{8}$ requires the use of a specialist slicing device and also involves slicing of the whole prostate at the time of fresh tissue sampling with shaves rather than cones made of the apex and basal margins. The use of shaved margins is discouraged in the RCPath dataset as distances to these margins cannot be accurately assessed. The majority of methods reviewed in this discussion were written before the RCPath dataset (second edition) for prostatic carcinoma was published in October 2009. ${ }^{18}$
Extraprostatic extension and margin positivity (among other factors) determine if patients enter trials or receive adjuvant therapy after surgery. The method described in this paper does not compromise assessment of either of these as the capsule is avoided during sampling and the margins remain intact with almost all of the prostate being submitted for routine histological analysis. Methods describing stripping of the capsule, such as that described by Bova et al, ${ }^{11}$ would not meet the current requirements of the RCPath dataset. ${ }^{18}$

Methods involving removal of large amounts of tissue for research also compromise routine reporting. The method recently described by Morrison et al ${ }^{12}$ takes every other slice for research. The RCPath dataset states that it is preferable to embed the entire prostate as in a study of 1383 patients, those with negative margins using step sectioning of the entire specimen had a lower risk of progression than patients whose specimens were partially sampled. ${ }^{21}$ In the Morrison et al method, although the entire capsule is examined for diagnosis, it is obtained by being stripped from the rest of the slice, which is harvested for research tissue (and divided into quadrants) hampering assessment of extraprostatic extension. The authors also state in their paper that the method is time consuming.

Bertlisson et al ${ }^{10}$ describe a method where a whole slice of prostate is taken intact for research. This technique requires a specialised plastic rack and cryoclamp to cut and freeze the slice. Specimens are removed from the slice with a drilling device. The slice is not available for diagnosis, but it is only taken at $2 \mathrm{~mm}$ thickness. To locate the tumour for research, tissue from adjacent paraffin blocks is examined and matched to the frozen slice by transposing an image. The major barrier to widespread use of this protocol is the equipment required and labour-intensive method to identify and obtain samples of tumour, compared to our method in which after routine histological reporting, the likely nature of the banked samples is known.

In comparison with other described methods, the method described here compares favourably on the number of cases in which tumour has been successfully sampled at $88 \%$. Others who used methods which were not reliant on visualisation of the tumour for sampling quoted rates of $75 \%{ }^{8}$ and $72 \% .{ }^{12}$ Lower positive rates were quoted by those using methods in which the tumour needed to be macroscopically evident, for example, $57 \%$ and $63 \%{ }^{13} 14$

In conclusion, we describe a systematic, rapid method of fresh tissue sampling which balances the need for accurate routine histopathological reporting with the need to provide good-quality fresh samples for research that are able to supply quickly the detailed morphological information necessary to meet the needs of researchers.

\section{What this study adds}

This paper describes a method of sampling for fresh radical prostatectomy specimens designed specifically to meet the needs of current research into prostatic carcinoma in that tissue is biobanked from the anterior, peripheral and transition zones with morphological confirmation not just of the nature of the tissue (benign/malignant), but also of Gleason grade and tumour stage. The protocol is logical, systematic and requires no specialised equipment. 


\section{Take-home messages}

- Newly emerging research into prostate cancer requires increasingly complex information about samples such as zone of origin, Gleason grade and stage whilst not compromising histological reporting to the standards set by the Royal College of Pathologists.

- This article reviews previously described methods of fresh tissue sampling from radical prostatectomy specimens for research and describes a method used in Oxford which is simple, logical and cost effective.

- The method of fresh radical prostatectomy sampling described in this article involves punch biopsies sampled from a slice approximately $8 \mathrm{~mm}$ superior to the apex.

Acknowledgements Professor Lars Egevad, Karolinska Institute,Stockholm, Sweden (with whom we are in a long established research collaboration with), for ongoing help with our methods of radical prostatectomy sampling for research.

We acknowledge the contribution to this study made by the Oxford Centre for Histopathology Research and the Oxford Radcliffe Biobank, which are supported by the NIHR Oxford Biomedical Research Centre.

Contributors All of the authors of this paper (PSG, ISDR, LB, RP, AYW, FCH, CV) were involved in design and conception of the method and drafting and reviewing the manuscript. All approved the final manuscript. There is no one else who should have been included as an author who has been omitted.

Funding The NIHR Biomedical Research Centre (BRC), Oxford funds research time for $P G, L B$ and $C V$.

Competing interests None.

Ethics approval NRES.

Provenance and peer review Commissioned; internally peer reviewed.

\section{REFERENCES}

1. http://info.cancerresearchuk.org/cancerstats/types/prostate/incidenceo/ (accessed 1 Jun 2012).

2. Curtis C, Shah SP, Chin S-F, et al. The genomic and transcriptional architecture of 2000 breast tumours. Nature 2012;486:346-52.

3. Ascierto PA, Kirkwood JM, Grob J-J, et al. The role of BRAF V600 mutation in melanoma. J Trans/ Med 2012;10:85.
4. Gerlinger M, Rowan AJ, Horswell S, et al. Intratumor heterogeneity and branched evolution revealed by multiregion sequencing. $N$ Eng J Med 2012;366:883-92.

5. Falzarano SM, Navas M, Simmerman $\mathrm{K}$, et al. ERG rearrangement is present in a subset of transition zone prostatic tumors. Mod Pathol 2010;23 1499-506.

6. Al-Ahmadie HA, Tickoo SK, Olgac S, et al. Anterior predominant prostatic tumours: zone of origin and pathologic outcomes at radical prostatectomy. Am J Surg Pathol 2008;32:229-35.

7. Egevad L. Handling of radical prostatectomy specimens. Histopathology 2012;60 $118-24$.

8. Jhavar SG, Fisher C, Jackson A, et al. Processing of radical prostatectomy specimens for correlation of data from histopathological, molecular biological and radiological studies: a new whole organ technique. J Clin Pathol 2005;58:504-8.

9. Egevad L, Engstrom K, Busch C. A new method for handling radical prostatectomies enabling fresh tissue harvesting, whole mount sections, and landmarks for alignment of sections. J Urol Pathol 1998;9:17-28.

10. Bertlisson $\mathbf{H}$, Angelsen A, Viset $\mathrm{T}$, et al. A new method to provide a fresh frozen prostate slice suitable for gene expression study and MR spectroscopy. Prostate 2011;1:461-9.

11. Bova GS, Fox WM, Epstein JL. Methods of radical prostatectomy specimen processing: a novel technique for harvesting fresh prostate cancer tissue and review of processing techniques. Mod Pathol 1993;6:201-7.

12. Morrison C, Cheney R, Johnson CS, et al. Central quadrant procurement of radical prostatectomy specimens. Prostate 2009;69:770-3.

13. Furman J, Murphy WM, Rice L, et al. Prostatectomy tissue for research. Am J Clin Pathol 1998;110:4-9.

14. Riddick ACP, Barker $C$, Bass $R$, et al. Banking of fresh-frozen prostate tissue: methods, validation and use. BJU 2003;91:315-24.

15. Renshaw AA. Correlation of gross morphologic features with histologic features in radical prostatectomy specimens. Am J Clin Pathol 1998;110:38-42.

16. Hoedemaeker RF, Ruijter ETG, Ruizeveld-de Winter JA, et al. Processing radical prostatectomy specimens. J Urol Pathol 1998;9:211-22.

17. Dev H, Rickman D, Sooriakumaran P, et al. Biobanking after robotic assisted radical prostatectomy: a quality assessment of providing prostate tissue for RNA studies. J Transl Med 2011;9:121.

18. Royal College of Pathologists. Dataset for histopathology reports for prostatic carcinoma. 2nd edn. RCPath, October 2009

19. Wheeler TM, Lebovitz RM. Fresh tissue harvest for research from prostatectomy specimens. Prostate 1994;25:274-9.

20. Warren AY, Whitaker HC, Haynes B, et al. Method for sampling fresh tissue for research which preserves pathological data in radical prostatectomy. Prostate 2012 , Published online 16th July 2012. 10.1002/pros.22556.

21. Grossfeld GD, Chang JJ, Broering JM, et al. Does the completeness of prostate sampling predict outcome for patients undergoing radical prostatectomy? Data from the CAPSURE database. Urology 2000;56:430-5. 